\title{
Combined femtosecond laser-assisted intracorneal ring segment implantation and corneal collagen cross-linking for correction of keratoconus
}

This article was published in the following Dove Press journal:

Clinical Ophthalmology

22 March 2016

Number of times this article has been viewed

\author{
Osama Ibrahim' \\ Ahmed Elmassry' \\ Amr Said ${ }^{1,2}$ \\ Moones Abdalla ${ }^{2}$ \\ Hazem El Hennawi' \\ Ihab Osman' \\ 'Department of Ophthalmology, \\ Alexandria University, Alexandria, \\ Egypt; ${ }^{2}$ Roayah Vision Correction \\ Center, Alexandria, Egypt
}

Correspondence: Amr Said Roayah Vision Correction Center, 52 Tout Ankh Amoun Street, Sidi Gaber Buildings, Elzahraa Building, Alexandria 21532, Egypt

Tel +201226154154

Fax +2034247470

Email docamrol@hotmail.com
Purpose: To assess the safety, predictability, and effectiveness of Keraring intrastromal corneal ring segments (ICRS) insertion assisted by femtosecond laser and corneal collagen cross-linking (CXL) for keratoconus correction.

Patients and methods: In this prospective, noncomparative, and interventional case series, 160 eyes of 100 adult keratoconus patients with poor best-corrected visual acuity (BCVA) (less than 0.7) and intolerance to contact lens wear were included. Patients underwent femtosecond laser-assisted placement of ICRS and CXL. All patients were examined for a complete ophthalmological test: uncorrected visual acuity (UCVA), BCVA, spherical equivalent, keratometry (K1-flat and K2-steep), pachymetry, and Scheimpflug imaging with the Pentacam at 1 week and at 1,3, and 6 months postoperatively.

Results: At 6 months, a significant difference was observed $(P<0.001)$ in mean UCVA and BCVA from $0.92 \pm 0.677$ and $0.42 \pm 0.600 \log$ MAR preoperatively to $0.20 \pm 0.568$ and $0.119 \pm 0.619$ $\log$ MAR, respectively. Mean spherical equivalent refractions were significantly lower $(P<0.001)$ at 6 months. Mean keratometry $(\mathrm{K})$ also significantly reduced $(P<0.001)$ from $50.93 \pm 5.53 \mathrm{D}$ (K1-flat) and 55.37 $\pm 5.76 \mathrm{D}$ (K2-steep) to $47.32 \pm 4.61$ and $51.08 \pm 5.38 \mathrm{D}$, respectively. In terms of pachymetry, no significant difference was observed preoperatively versus postoperatively $(P=1.000)$.

Conclusion: Keraring ICRS insertion assisted by femtosecond laser and corneal CXL provided significant improvement in visual acuity, spherical equivalent, and keratometry, which suggests that it may be effective, safe, and predictable for keratoconus correction.

Keywords: keratometry, pachymetry, keratectesia, Pentacam, irregular astigmatism

\section{Introduction}

Keratoconus is a noninflammatory, often inherited, chronic, most common primary corneal ectasia, which often includes progressive steepening, thinning, and apical scarring as prominent features. ${ }^{1}$ These corneal changes usually manifest as decreased visual acuity, progressive myopia, and irregular astigmatism. Appraisals vary; however, the annual estimated incidence is two in 100,000 with a prevalence of 54.5 in $100,000 .^{1-3}$

Contact lens-intolerant keratoconus patients without central scarring who have mild or moderate disease may be candidates for intracorneal ring segments (ICRS) insertion. Intracorneal rings were first used to correct low myopia and have more recently been studied for use in keratoconus to induce flattening of the cornea in order to delay, if not to avoid, corneal grafting. ${ }^{4}$ The segments induce an "arc-shortening effect" on the corneal lamellae and flatten the corneal center, as well as shift the corneal apex to a more central position that improves contact lens fit and comfort, thereby optimizing 
best-corrected visual acuity (BCVA). ${ }^{5}$ The ideal candidates for ICRS have moderate spherical equivalents and average keratometry readings of less than $65 \mathrm{D}$.

The high accuracy cuts to the corneal tissue demonstrated by femtosecond lasers in refractive surgery have generated new inquiry on their application into tunnel creation for precision ICRS insertion. The advantages of femtosecond channel creation over mechanical approaches are the following: a more uniform dissection is created; the results and ICRS placement are more consistent; and because it is minimally invasive, there is less patient discomfort and quicker recovery. ${ }^{6}$

Corneal collagen cross-linking (CXL) slows down the progression of keratectasia. The procedure uses ultraviolet (UV) light and riboflavin to strengthen the stromal collagen. The irradiation of riboflavin results in chemical reactions that create covalent bonds that bridge amino groups of the stromal collagen fibrils. ${ }^{7}$ The principal effects of CXL are localized to the anterior $300 \mu \mathrm{m}$ of the stroma. Individuals with corneas thinner than $400 \mu \mathrm{m}$ should not undergo CXL because of possible endothelial cell damage. ${ }^{8}$ Several studies have demonstrated that CXL is effective in halting the progression of ectasia in keratoconus..$^{-11}$

There have been few studies on the outcomes of combined ICRS and CXL, but they have reported that CXL, when combined with ICRS insertion in keratoconus patients, has an additive effect on spherical equivalent refractions and keratometry. ${ }^{12-14}$ In reports of applied femtosecond laser technology to assist in ICRS insertion, findings recommended that channel dissection using femtosecond and ICRS implantation should be performed before or concurrent with CXL as ICRS-induced flattening of the cornea may be affected by CXL-induced corneal stiffness if applied after CXL. ${ }^{15}$

In this case series, we present the results of femtosecond laser-assisted placement of ICRS (Keraring, Mediphacos, Belo Horizonte, Brazil) and subsequent CXL in the same setting for visual correction of patients with keratoconus.

\section{Patients and methods}

One hundred adult patients with keratoconus were included in this prospective, noncomparative, interventional case series between July 2011 and March 2013 at Roayah Vision Correction Center, Alexandria, Egypt. Inclusion criteria involving patients aged 18-40 years who underwent CXL consisted of progressive keratoconus patients (those with $1 \mathrm{D}$ increase in steepest keratometric reading over a 6-month period as documented with the Pentacam [Allegro Oculyzer, WaveLight AG, Erlangen, Germany], poor BCVA [less than 0.7], intolerance to contact lens wear, clear central cornea, and peripheral corneal thickness of more than $450 \mu \mathrm{m}$ at the planned incision sites). Exclusion criteria were corneal haze or opacity, severe keratconus (keratometric values of $>65.0 \mathrm{D}$ ), acute hydrops, and the presence of active or recent ocular infection or inflammation.

Prior to the study's commencement, the scope of subject participation was clearly explained to all patients, and they gave written informed consent in accordance with institutional guidelines according to the Declaration of Helsinki. The ethics committee of Alexandria University, Faculty of Medicine, approved this study.

Preoperatively, all patients underwent a complete ophthalmic test that included UCVA, manifest refraction, BCVA, and slit-lamp examination to exclude corneal opacity or inflammation, a fundus examination to report any posterior segment abnormalities, Scheimpflug imaging with Pentacam to measure keratometric readings and corneal thickness at the thinnest region of the cornea, and anterior segment optical coherence tomography (OCT; Visante, Carl Zeiss Meditec AG, Jena, Germany) to measure corneal thickness of identified incision points.

To plan for ICRS (Keraring) insertion, the nomogram provided by the manufacturer, Mediphacos, was used to select the proper ring segments (Keraring calculation guidelines).

All cases were operated on by the same surgeon (Dr Osama Ibrahim). The procedure was performed under topical anesthesia (benoxinate eye drops $\left[\right.$ Benox $^{\circledR}$, Epico, Inc., Cairo, Egypt] given three to five times every 5 minutes). Sterilization of both eyes was performed with povidoneiodine, followed by draping, and application of a speculum to the eye to be treated.

Patients underwent an ultrafast $\left(10^{-15} / \mathrm{s}\right)$ femtosecond laser procedure (VisuMax ${ }^{\circledR}$, Carl Zeiss Meditec AG) to create tunnels and incisions at a frequency of $500 \mathrm{kHz}$, a wavelength of approximately $1,040 \mathrm{~nm}$ and a $1 \mu \mathrm{m}$ spot size. With the VisuMax ${ }^{\circledR}$ software, it is possible to select the depth and position of the tunnels ( $80 \%$ of corneal thickness; parallel to the cornea's posterior surface).

Sterile VisuMax ${ }^{\circledR}$ Disposable Treatment Packs (Carl Zeiss Meditec AG) consisting of a contact glass and hose were utilized. The sterile contact glass was positioned on the laser aperture, and the hose was connected to a filter in the unit. The curved interface of the contact glass was docked to the cornea by means of suction pressure, and it was inspected for proper centration. The suction diameter chosen was slightly less than the white-to-white corneal diameter measured by corneal topography. The disposable 
contact glass minimally applanates the cornea, maintaining a precise focal distance between the laser emission aperture and the desired focal point, forming an ICRS tunnel of desired depth and geometry cut within the stroma.

The steepest corneal topographic axis was selected as the incision point. In all cases, two incisions were created, except when a 210 -arc ICRS was used, wherein only one incision was made. The number of tunnels depended on the desired number of segments to be inserted. One tunnel, usually an inferior one, was created if one segment was planned for use, while two tunnels were created if two segments were to be inserted. Each tunnel was approximately $4.95 \mathrm{~mm}$ in inner diameter and $6.44 \mathrm{~mm}$ in outer diameter (Figure 1A). Energy used was less than $1 \mathrm{~mJ}$, with a procedure time of approximately 18 seconds for the femtosecond laser to create all tunnels and incisions. Upon completion of incisions and tunnels, the patient was automatically released from the laser system contact glass. The access incision and the tunnel were tested to ensure their patency.

The Keraring ICRS were placed after channel creation under full aseptic technique with the aid of Keraring forceps and a Sinskey hook through dialing holes at both ends of the segments. Each segment was centered in the middle of its tunnel, equidistant from both incisions. When a single segment was inserted, it was inserted inferiorly, while twosegment implantation required the largest one to be inserted inferiorly.

Questionnaires about patient satisfaction were answered by all patients at the sixth follow-up month. These included questions about improvement in quality of vision, improvement in far and near vision, dry eye symptoms, and night vision problems (glare or halos).

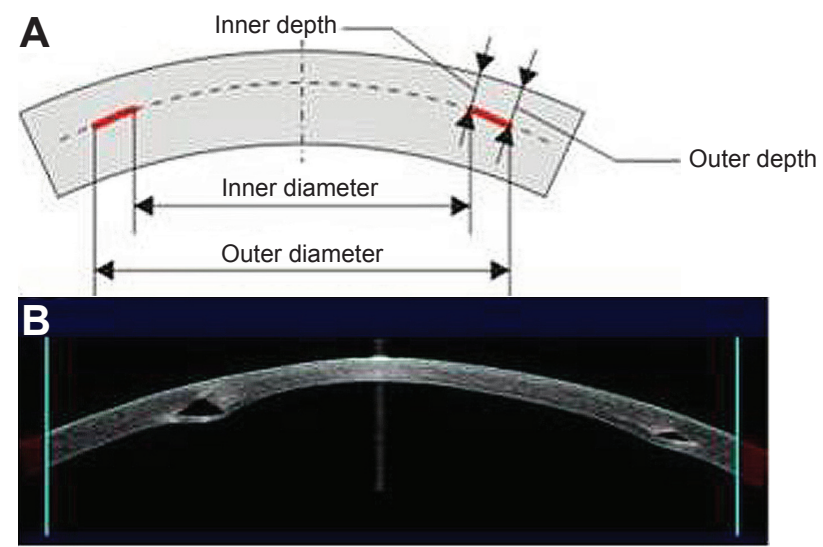

Figure I (A) Side view of the section of the cornea with tunnel incision sections. (B) Postoperative anterior segment OCT images showing ICRS in place. Abbreviations: OCT, optical coherence tomography; ICRS, intrastromal corneal ring segments.

\section{Corneal collagen cross-linking}

Corneal collagen CXL was performed in all patients. In our case series, only those with progressive keratoconus (defined as a $1 \mathrm{D}$ increase in steepest keratometric reading over a period of 6 months as documented with Pentacam) were included in the study.

For CXL, the central corneal epithelium was removed to allow better diffusion of riboflavin into the stroma. Thirty minutes before irradiation, $0.1 \%$ riboflavin solution was applied and reapplied every 5 minutes thereafter. A CXL device (PESCHKE Trade CCL-VARIO Cross-linking; Swissmed, Gdańsk, Poland) that emits a UVA wavelength of $370 \mathrm{~nm}$, and an irradiance of $18 \mathrm{~mW} / \mathrm{cm}^{2}$ for 5 minutes (equivalent to $5,400 \mathrm{~mJ}$ of the total energy dosage) at $5 \pm 0.5 \mathrm{~cm}$ distance, was used for irradiation. Contact lenses were placed at the end of procedure.

\section{Postoperative treatment}

The topical antibiotic moxifloxacin (Vigamox, Alcon Laboratories, Inc., Fort Worth, TX, USA), steroids (prednisolone; PredForte, Allergan, Westport, Ireland), and artificial tears were used postoperatively. The artificial eye drops were used every hour during the first day, and then every 4 hours for 1 month. The antibiotic dose timing was every hour for the first day, which then decreased to once every 4 hours for 10 days. The systemic analgesic indomethacin (Cataflam, Novartis, Basel, Switzerland) was prescribed to manage pain due to CXL. Patients were instructed to avoid eye rubbing. Contact lenses were removed once epithelial healing was complete. This usually occurred on the third day after surgery. All patients were examined for a complete ophthalmological test, including BCVA, refraction, and Scheimpflug imaging with the Pentacam at 1, 3, and 6 months, postoperatively. Anterior segment OCT was done only once during follow-up at 1 week to detect ICRS depth and position (Figure 1B). All complications, if any, were documented.

\section{Statistical analysis}

Data were analyzed using the Predictive Analytics Software (PASW Statistics version 18 for Windows, Quarry Bay, Hong Kong). Quantitative data were described using median, minimum, and maximum, as well as mean and standard deviation. Comparison between different periods was assessed using an analysis of variance test with repeated measures and Bonferroni correction. Significance test results were quoted as two-tailed probabilities. The significance of the obtained results was determined at the $5 \%$ level. 


\section{Results}

This study included 160 eyes (100 keratoconus patients). The patient age range was $18-40$ years (mean $\pm \mathrm{SD} ; 21.77 \pm 6.78$ ). There were 43 males (78 eyes) and 57 females (82 eyes). Sixty patients had bilateral ICRS implantation.

\section{Intraoperative and postoperative complications}

No intraoperative complication was reported, except for difficult insertion in one case that had 210-arc segments to be placed. However, a successful implantation proceeded when it was replaced by another shorter segment in the same setting of the primary operation, without the need to use a mechanical spreader. There was also no need for sutures in any of the operated cases. In three eyes, intraoperative suction loss due to patient movement occurred. Suction was achieved again in the same setting with no resultant decentration or perforation. Excellent corneal tolerance was observed in all eyes, with no keratitis, extrusion, migration, or vascularization around the incision or the tunnels. In addition, neither corneal ulcers nor stromal necrosis superficial to the segment was reported. In one patient, the ICRS broke while still in place. This patient showed no improvement in BCVA. He did show improvement in UCVA by four lines (from 20/200 to $20 / 80$ ). The patient was satisfied with the surgical results and refused to have the segment removed. No postoperative infection or corneal melting occurred during follow-up in any of the cases. With the use of anterior segment OCT, three eyes were found to have ICRS inverted upside down in the corneal tunnel, but no further corrections were necessary as no impact on the visual outcome was noticed.

\section{Visual outcomes}

Table 1 summarizes the preoperative and postoperative outcomes. The UCVA was significantly better $(P<0.001)$ than preoperative measurements at all follow-up visits. The mean UCVA improved from 0.92 $\pm 0.677 \log$ MAR preoperatively to $0.200 \pm 0.568 \log$ MAR at 6 months postoperatively. Comparison of UCVA at different follow-up periods did not show any statistically significant difference.

The BCVA was $0.5 \mathrm{D}$ or better in 80 out of 160 eyes $(50 \%)$ at 1 month, in 100 out of 160 eyes $(60 \%)$ at 3 months, and in 120 out of 160 eyes (75\%) at 6 months. The improvement in BCVA increased from $0.42 \pm 0.600 \log$ MAR preoperatively to $0.119 \pm 0.619 \log$ MAR after 6 months postoperatively, and $75 \%$ of eyes showed improvement in two lines or more. No eye lost BCVA lines.

In all patients, marked improvement in spherical equivalent was observed. Mean spherical equivalent refractions were significantly lower $(P<0.001)$ at 6 months, a reduction from $-9.28 \pm 4.70$ to $-5.82 \pm 2.9 \mathrm{D}$.

The overall patient satisfaction according to the applied questionnaire at the sixth follow-up visit was $91 \%$. The most common cause of poor satisfaction was noticed to be severe dry eye symptoms (six patients), followed by night vision problems (three patients).

\section{Discussion}

In our case series, we present the findings from 100 keratoconus patients who underwent Keraring ICRS insertion assisted by femtosecond laser and CXL. After 6 months of follow-up, the patients demonstrated significant improvement $(P<0.001)$ in UCVA and BCVA, as well as a significant reduction $(P<0.001)$ in spherical equivalent refraction. In addition, mean keratometry readings were also significantly reduced $(P<0.001)$. These patients did not experience any serious intraoperative or postoperative complications.

Most studies seem to indicate that the best indications for ICRS are the presence of mild-to-moderate keratoconus with a clear optical zone and intolerance to contact lens wear. ${ }^{5}$

All patients in this study had moderate keratoconus with clear central corneas, and all eyes demonstrated excellent corneal tolerance without extrusion, migration, or

Table I Summary of preoperative and postoperative outcomes

\begin{tabular}{lllll}
\hline Outcome & Mean \pm SD & & & \\
\cline { 2 - 5 } & Preoperative & Postoperative (I month) & Postoperative (3 months) & Postoperative (6 months) \\
\hline UCVA (in logMAR) & $0.92 \pm 0.677$ & $0.20 \pm 0.49(P<0.00 \mathrm{I})$ & $0.22 \pm 0.53(P<0.00 \mathrm{I})$ & $0.20 \pm 0.568(P<0.00 \mathrm{I})$ \\
BCVA (in logMAR) & $0.42 \pm 0.600$ & $0.119 \pm 0.43(P<0.00 \mathrm{I})$ & $0.09 \pm 0.657(P<0.00 \mathrm{I})$ & $0.1 \mathrm{I} \pm 0.6 \mathrm{I}(P<0.00 \mathrm{I})$ \\
Spherical equivalent & $-9.28 \pm 4.7 \mathrm{D}$ & $-5.28 \pm 2.12 \mathrm{D}(P<0.00 \mathrm{I})$ & $-5.34 \pm 2.82 \mathrm{D}(P<0.00 \mathrm{I})$ & $-5.82 \pm 2.90 \mathrm{D}(P<0.00 \mathrm{I})$ \\
KI & $50.93 \pm 5.53 \mathrm{D}$ & $48.19 \pm 4.93 \mathrm{D}(P<0.033)$ & $46.77 \pm 4.87 \mathrm{D}(P<0.005)$ & $47.32 \pm 4.6 \mathrm{I} \mathrm{D}(P<0.05)$ \\
K2 & $55.37 \pm 5.76 \mathrm{D}$ & $50.99 \pm 5.48 \mathrm{D}(P<0.00 \mathrm{I})$ & $50.88 \pm 5.72 \mathrm{D}(P<0.00 \mathrm{I})$ & $5 \mathrm{I} .08 \pm 2.1 \mathrm{D}(P<0.00 \mathrm{I})$ \\
Pachymetry $(\mu \mathrm{m})$ & $43 \mathrm{I} .19 \pm 43.20$ & $418.34 \pm 58.52(P=\mathrm{I} .000)$ & $402.2 \mathrm{I} \pm 85.04(P$-value I.000) & $422.84 \pm 58.14(P$-value I.000) \\
\hline
\end{tabular}

Abbreviations: UCVA, uncorrected visual acuity; BCVA, best-corrected visual acuity; KI, flat keratometry; K2, steep keratometry. 
vascularization around the incision or the tunnels. In addition, neither corneal ulcers nor stromal necrosis superficial to the segment was reported. One patient was found to have one of his ICRS broken while it still remained in place. This patient showed improvement in his UCVA although he maintained his preoperative BCVA.

The results of this case series demonstrate that spherical equivalent error and astigmatism (refractive and keratometric) were reduced in all cases. Compared to baseline, the topographic corneal shape (size and height of the cone) improved in all patients. An approximate decrease of $3 \mathrm{D}$ was observed in mean keratometry, and the keratometric astigmatism decreased during the 6-month follow-up. The majority of patients (91 out of 100) were satisfied with their surgical results.

Chan et al $^{16}$ confirmed that combined ICRS and CXL is more effective compared to ICRS alone in patients with keratoconus. They found that patients who underwent the combined procedure demonstrated significantly greater reduction in cylinder, steep and average keratometry, and the lower-upper ratio - a topographic measure of the degree of keratoconus. ${ }^{16}$

Coskunseven et $\mathrm{al}^{17}$ investigated the sequences of combined ICRS implantation and UV/riboflavin-mediated CXL in patients with progressive keratoconus. This prospective comparative randomized consecutive study compared the two sequence options (CXL first, followed by ICRS implantation versus ICRS implantation first, followed by CXL). The study findings demonstrated that the second sequence option (ICRS implantation followed by CXL) resulted in greater improvement in keratoconus, compared to the first sequence option (CXL followed by ICRS implantation). A stiffer cornea previously treated by CXL exhibits a decreased flattening effect of ICRS implantation, hence reining in its effect and reducing the maximum flattening potential. Therefore, to achieve optimal effect, ICRS implantation should be performed first, so that the segments can reshape the cornea without constraint. The CXL treatment can then follow to additionally flatten the cornea and to stabilize its biomechanics. ${ }^{17}$ In addition, Ertan and Colin ${ }^{18}$ recommended intrastromal ring implantation combined with corneal CXL to prevent keratoconus progression and amplify the ring segments' flattening effect.

We are also aware of two case series that investigated same day CXL and ICRS placement. Kilic et al ${ }^{19}$ followed a large series of 131 eyes of 105 patients for a mean period of 7 months and, similar to our study, they found improved UDVA, CDVA, refraction, and keratometry.
Similarly, Saelens et $\mathrm{al}^{20}$ followed a smaller series of seven patients for a year, and the reported results are similar to our study - a significant improvement in BCVA and UCVA, a significant reduction in mean spherical equivalent of $3.5 \mathrm{D}$, as well as lower mean keratometry values. They also found, as we did, that the thinnest pachymetry measures did not change significantly after treatment. ${ }^{20}$

\section{Conclusion}

In conclusion, Keraring ICRS insertion assisted by femtosecond laser and corneal CXL provided significant improvement in visual acuity, spherical equivalent, and keratometry results, which seems to suggest that it may be an effective, favorable treatment for the correction of keratoconus.

\section{Disclosure}

The authors have no financial or proprietary interest in any materials or methods presented herein. Dr Ibrahim is a consultant for Carl Zeiss Meditec. The authors report no other conflicts of interest in this work.

\section{References}

1. Rabinowitz YS. Keratoconus. Surv Ophthalmol. 1998;42:297-319.

2. Krachmer JH, Feder RS, Belin MW. Keratoconus and related noninflammatory corneal thinning disorders. Surv Ophthalmol. 1984;28: 293-322.

3. Kennedy RH, Bourne WM, Dyer JA. A 48-year clinical and epidemiologic study of keratoconus. Am J Ophthalmol. 1986;101:267-273.

4. Colin J, Cochener B, Savary G, Malet F. Correcting keratoconus with intracorneal rings. J Cataract Refract Surg. 2000;26(8):1117-1122.

5. Coskunseven E, Kymionis GD, Tsiklis NS, et al. Complications of intrastromal corneal ring segment implantation using a femtosecond laser for channel creation: a survey of 850 eyes with keratoconus. Acta Ophthalmol. 2011;89:54-57.

6. Ratkay-Traub I, Ferincz IE, Juhasz T, Kurtz RM, Krueger RR. First clinical results with the femtosecond neodynium-glass laser in refractive surgery. J Refract Surg. 2003;19:94-103.

7. Wollensak G, Spoerl E, Seiler T. Stress-strain measurements of human and porcine corneas after riboflavin-ultraviolet-A-induced cross-linking. J Cataract Refract Surg. 2003;29:1780-1785.

8. Goldich Y, Marcovich AL, Barkana Y, Avni I, Zadok D. Safety of corneal collagen cross-linking with UV-A and riboflavin in progressive keratoconus. Cornea. 2010;29:409-411.

9. Lamy R, Netto CF, Reis RG, et al. Effects of corneal cross-linking on contrast sensitivity, visual acuity, and corneal topography in patients with keratoconus. Cornea. 2013;32(5):591-596.

10. Caporossi A, Mazzotta C, Baiocchi S, Caporossi T. Long-term results of riboflavin ultraviolet a corneal collagen cross-linking for keratoconus in Italy: the Siena eye cross study. Am J Ophthalmol. 2010;149(4): 585-593.

11. Wollensak G, Spoerl E, Seiler T. Riboflavin/ultraviolet-a-induced collagen crosslinking for the treatment of keratoconus. Am J Ophthalmol. 2003;135(5):620-627.

12. Alió JL, Artola A, Hassanein A, Haroun H, Galal A. One or 2 Intacs segments for the correction of keratoconus. J Cataract Refract Surg. 2005;31(5):943-953. 
13. El-Raggal TM. Sequential versus concurrent KERARINGS insertion and corneal collagen cross-linking for keratoconus. Br J Ophthalmol. 2011;95(1):37-41.

14. El Awady H, Shawky M, Ghanem AA. Evaluation of collagen crosslinking in keratoconus eyes with Kera intracorneal ring implantation. Eur J Ophthalmol. 2012;22(Supp1 7):S62-S68.

15. El-Raggal TM. Effect of corneal collagen crosslinking on femtosecond laser channel creation for intrastromal corneal ring segment implantation in keratoconus. J Cataract Refract Surg. 2011;37(4):701-705.

16. Chan CC, Sharma M, Wachler BS. Effect of inferior-segment Intacs with and without C3-R on keratoconus. J Cataract Refract Surg. 2007; 33(1):75-80.

17. Coskunseven E, Jankov MR II, Hafezi F, Atun S, Arslan E, Kymionis GD. Effect of treatment sequence in combined intrastromal corneal rings and corneal collagen crosslinking for keratoconus. J Cataract Refract Surg. 2009;35:2084-2091.
18. Ertan A, Colin J. Intracorneal rings for keratoconus and keratectasia. J Cataract Refract Surg. 2007;33(7):1303-1314.

19. Kilic A, Kamburoglu G, Akinci A. Riboflavin injection into the corneal channel for combined collagen crosslinking and intrastromal corneal ring segment implantation. J Cataract Refract Surg. 2012;38:878-883.

20. Saelens IE, Bartels MC, Bleyen I, Van Rij G. Refractive, topographic, and visual outcomes of same-day corneal cross-linking with Ferrara intracorneal ring segments in patients with progressive keratoconus. Cornea. 2011;30:1406-1408.
Clinical Ophthalmology

\section{Publish your work in this journal}

Clinical Ophthalmology is an international, peer-reviewed journal covering all subspecialties within ophthalmology. Key topics include: Optometry; Visual science; Pharmacology and drug therapy in eye diseases; Basic Sciences; Primary and Secondary eye care; Patient Safety and Quality of Care Improvements. This journal is indexed on

\section{Dovepress}

PubMed Central and CAS, and is the official journal of The Society of Clinical Ophthalmology (SCO). The manuscript management system is completely online and includes a very quick and fair peer-review system, which is all easy to use. Visit http://www.dovepress.com/ testimonials.php to read real quotes from published authors. 